\title{
The Mode of Host Resistance to Plasmopara viticola Infection of Grapevines
}

\author{
Ying Yu, Yali Zhang, Ling Yin, and Jiang Lu
}

First, second, third, and fourth authors: College of Food Science and Nutritional Engineering, China Agricultural University, Beijing, 100083, China; and fourth author: Center for Viticulture and Small Fruit Research, Florida A\&M University, Tallahassee, FL 32317. Y. Yu and Y. Zhang contributed equally to this work.

Accepted for publication 26 July 2012.

\begin{abstract}
Yu, Y., Zhang, Y., Yin, L., and Lu, J. 2012. The mode of host resistance to Plasmopara viticola infection of grapevines. Phytopathology 102:10941101.

The resistance and susceptibility of grapevines to downy mildew (DM) disease caused by Plasmopara viticola were compared among different cultivars/accessions belonging to Vitis vinifera, V. rotundifolia, and 10 oriental Vitis species. After inoculation with $P$. viticola pathogen, no symptom was found in $V$. rotundifolia grapevines at all, while oriental species $V$. davidii and $V$. piasezkii, like $V$. vinifera, were susceptible to DM disease. The other eight oriental Vitis species showed various resistance levels to DM disease. Intraspecific resistant variations were

various time courses after pathogen infection on grape leaves. $P$. viticola hyphae were not observed in $V$. rotundifolia cultivars, while symptoms with varying degrees of severity were observed among the Euvitis species. In general, the DM resistant oriental species showed a slower development of hypha and less formation of haustoria than DM susceptible $V$. vinifera grapevines. Cells with distinctive fluorescence were observed in $V$. rotundifolia and the oriental species $V$. pseudoreticulata, and callose deposits were observed in V. rotundifolia, V. pseudoreticulata, and $V$. amurensis grapevines. Based on the results of morphological observations and microscopy studies, we concluded that there were five levels of grapevine resistance to $P$. viticola pathogen: (i) immune, (ii) extremely resistant, (iii) resistant, (iv) partly resistant, and (v) susceptible.
\end{abstract} also observed in $V$. amurensis. Microscopy studies were conducted on
Downy mildew (DM) is one of the most destructive grapevine diseases worldwide, particularly in those viticulture areas with warm and humid climate conditions (4). DM is caused by a causal agent Plasmopara viticola (Berk. \& M.A. Curtis) Berl \& De Toni in Sacc., belonging to the oomycetes, family Peronosporaceae (21). P. viticola is an obligate biotroph and thus requires the genus Vitis to remain alive in order to complete its life cycle. It cannot survive outside its host except as oospores. When inoculating onto the abaxial surface of a susceptible grape leaf, zoospores germinate to form a germ tube which grows through a stoma (9). Then hyphae develop with some branches and numerous haustoria, which could penetrate cell walls of the mesophyll (54). At last, sporangiophores emerge through the stomata where they expand into tree-shaped structures carrying the sporangia. In resistant species, the infection progress is slowed down, inhibited, or completely stopped.

$P$. viticola could cause extensive losses in grape yield as it can severely infect leaves, inflorescences, and fruit clusters. Variation in resistance to $P$. viticola among different Vitis species and cultivars has been reported $(1,4-6,55)$. In general, the European grapevines ( $V$. vinifera) are found to be susceptible to $P$. viticola in varying degrees, while the North American Vitis species exhibit resistance to this pathogen (1). The resistances of oriental Vitis species to the DM disease are variable. For example, some species, such as $V$. davidii var. cyanocarpa, $V$. pseudoreticulata, and $V$. yeshanensis, were found to be resistant to DM disease, while others, such as $V$. adstricta and $V$. hancockii, were suscepti-

Corresponding author: J. Lu; E-mail address: j.lu.cau@gmail.com

* The $\boldsymbol{e}$-Xtra logo stands for "electronic extra" and indicates that Figures 1 and 3 appear in color online.

http://dx.doi.org/10.1094/PHYTO-02-12-0028-R

(C) 2012 The American Phytopathological Society ble (55). Song et al. $(48,49)$ reported that DM resistance varied among different cultivars/clones of $V$. amurensis, the most cold hardy oriental Vitis species.

In grapevines, some common disease resistance mechanisms have been described $(20,23,27,33)$. The pathogen invasion could induce the defense reactions, including accumulation of metabolites such as stilbenes and pathogenesis-related proteins (32), formation of papillae or tyloses (31), lignification (29), synthesis of antifungal phytoalexins $(13,45)$, and often hypersensitive reaction (23). In biotrophic pathogens and plant interactions, the hypersensitive cell death could restrict the growth of the pathogen directly and also could stimulate the specificity defense reaction near the infected area and the systematic acquired resistance of plant (26). Feechan et al. (18) revealed that penetration resistance and programmed cell death (PCD) associated responses were employed by the different grapevines to limit E. necator infection. Callose and phenol depositions, and hypersensitive response-like cell death were also observed in resistant grapevines inoculated by $P$. viticola $(23,27)$, and these reactions work together to reduce pathogen growth in grapevines.

A comparison of $P$. viticola early development between a host system on grapevine leaf discs and a host-free system (36) demonstrated that host factors could regulate the early development of $P$. viticola. Jürges et al. (34) revealed that the pathogen $P$. viticola had different developing patterns on Vitis species with different DM resistance levels. In susceptible Vitis species, the pathogen can develop in the leaves easily, while in resistant host species, the invasive growth of $P$. viticola was delayed after attachment of zoospores and further development ceased before the mycelium filled the intercostal fields. Unger et al. (54) observed the differences of $P$. viticola haustoria formation between susceptible and resistant grapevines. The same phenomenon was also observed in cruciferous hosts affected by Hyaloperonospora parasitica (40). 
To better understand the interaction between grapevines and $P$. viticola pathogen, cultivars/accessions of different Vitis species representing very diverse genetic backgrounds were chosen in this study. Early infection events, host responses to the pathogen infection, and progress of the disease development were investigated. The main objectives of this investigation were to understand the mode of actions on resistant mechanisms to $P$. viticola infection and DM development on grapevines.

\section{MATERIALS AND METHODS}

Plant material and pathogen. Six cultivars of $V$. amurensis ('Changbaijiu', 'Shuangfeng', 'Shuanghong', 'Shuangyou', 'Tonghuasan', and 'Zuoshanyi'), two $V$. amurensis $\times V$. vinifera hybrids (VA-VV hybrids), 'Zuohongyi' and 'Zuoyouhong', four cultivars of $V$. vinifera ('Cabernet Sauvignon', 'Chardonnay', 'Thompson Seedless', and 'Yatomi Rosa') and three cultivars of V. rotundifolia ('Carlos', 'Fry', and 'Noble') were grown at China Agricultural University, Agricultural Experimental Station, Beijing. The other nine Chinese Vitis species ( $V$. adstricta, $V$. adenoclada, V. bellula, V. davidii, V. ficifolia, V. hancockii, V. piasezkii, V. pseudoreticulata, and $V$. quinquangularis) were grown in the National Grape Germplasm Repository in Zhengzhou Fruit Research Institute, Chinese Academy of Agricultural Science.

For the $P$. viticola inoculation experiments, none of the selected mixtures of $P$. viticola were obtained from the infected grape leaves in Beijing Academy of Agriculture and Forestry Sciences. The sporangia were collected in centrifuge tubes by a small paintbrush and resuspended in distilled water. The pathogenicity of these $P$. viticola mixtures was tested by inoculating them in the DM susceptible $V$. vinifera 'Cabernet Sauvignon' grapevine and the results indicated that their aggressiveness was quite high.

Inoculation procedure. Leaf discs were prepared according to Boso and Kassemeyer (4) with some modification. The third to fifth unfolded leaves from the apex of the 1-year-old branches were excised and placed in zip bags and immediately stored in ice box for transportation. Before inoculation, the excised leaves were rinsed with distilled water and carefully dried with household tissues. Leaf discs (11 $\mathrm{mm}$ in diameter) were obtained by a cork borer and placed (bottom side up) on water agar $(0.8 \%$ $\mathrm{wt} / \mathrm{vol})$ in petri dishes. Inoculation was done by applying $35-\mu \mathrm{l}$ droplets of the sporangial suspension $\left(10^{5}\right.$ sporangia/ml of deionized water) onto leaf discs. The concentration of sporangia was determined using a hemacytometer, and the droplets on leaf discs were removed $24 \mathrm{~h}$ postinoculation (hpi). The leaf discs in petri dishes were maintained in a culture chamber at $25 / 22^{\circ} \mathrm{C}$ with a photoperiod of $16 / 8 \mathrm{~h}$ (light/dark, respectively). Deionized water was applied as control.

Disease severity. Sixty discs of each genotype were inoculated as described above. Disease severity, disease incidence, and sporulation density on leaf discs were quantified 9 days postinoculation (dpi). Disease incidences were quantified by determining the number of discs with sporulation per total number of discs, as described by Boso and Kassemeyer (4). To score disease severity, the percentage of the leaf disc area exhibiting symptoms of sporulation was measured with LANE-1D analysis software (Beijing Sage Creation Science Co., Ltd., Beijing, China) and classified by the Desaymard standard ' 0 to 10 ' (14). The severity index (SI) was calculated using the following formula:

$$
\mathrm{SI}=\frac{\sum(\text { rank } \times \text { infected leaf disk numbers in that rank })}{\text { total leaf disc numbers } \times \text { highest rank }} \times 100
$$

The sporulation density was scored subjectively based on the scheme shown in Figure 1 , where $0=$ no $P$. viticola, $1=$ lower density, 2 = moderate density, $3=$ high density, and $4=$ very high density.

Data analysis. Data for each variable were analyzed by using analysis of variance to detect any significant differences. Post hoc comparison (Student Newman Keuls) was used to compare the level of resistance of each variety. Principal components analysis was performed for the results of all variables. All calculations were performed by using the SPSS 13.0 software (SPSS China).

Scanning electron microscopy. Leaf discs at 1 and 8 dpi were fixed with $2.5 \%$ glutaraldehyde in $0.2 \mathrm{M}$ phosphate buffer $(\mathrm{pH} 7)$ for overnight at $4^{\circ} \mathrm{C}$, washed three times with $0.2 \mathrm{M}$ phosphate buffer for $15 \mathrm{~min}$ at room temperature, dehydrated for $20 \mathrm{~min}$ each in a series of ethanol solutions (50\%-70\%-80\%-90\%-95\% and twice in 100\%), and immersed in isoamyl acetate for overnight. The tissues were then critically point dried (HCP-2 Critical Point Dryer) (Hitachi, Ltd., Japan) using liquid carbon dioxide and sputter coated with gold. The samples were examined using the HITACHI S-3000N SEM (Hitachi).

Fluorescence microscopy. Leaf discs were collected at distinct intervals from 1 to $8 \mathrm{dpi}$ and conserved at $-20^{\circ} \mathrm{C}$. For microscopy analysis, the specimens were treated according to the $\mathrm{KOH}$ aniline blue fluorescence method $(15,30)$ with some modifications. Leaf discs were clarified at $100^{\circ} \mathrm{C}$ in $1 \mathrm{M} \mathrm{KOH}$ for $30 \mathrm{~min}$, washed three times for $15 \mathrm{~min}$ each, and then soaked in $0.05 \%$ aniline blue (0.067 $\mathrm{M} \mathrm{K}_{2} \mathrm{HPO}_{4}$, pH 9 to 9.5) for $15 \mathrm{~min}$. Finally, the discs were examined under a fluorescence microscope (Olympus BX51 with an excitation filter $365 \mathrm{~nm}$ and an emission filter LP $420 \mathrm{~nm}$ ) (Olympus Corporation, Japan). The images were taken with an Olympus DP70 digital camera, and analyzed with the LANE-1D analysis and Photoshop software.

\section{RESULTS}

Variation in DM severity among Vitis species. Inoculation of $P$. viticola on leaf discs among the grape species/cultivars or accessions showed different levels of resistance and susceptibility
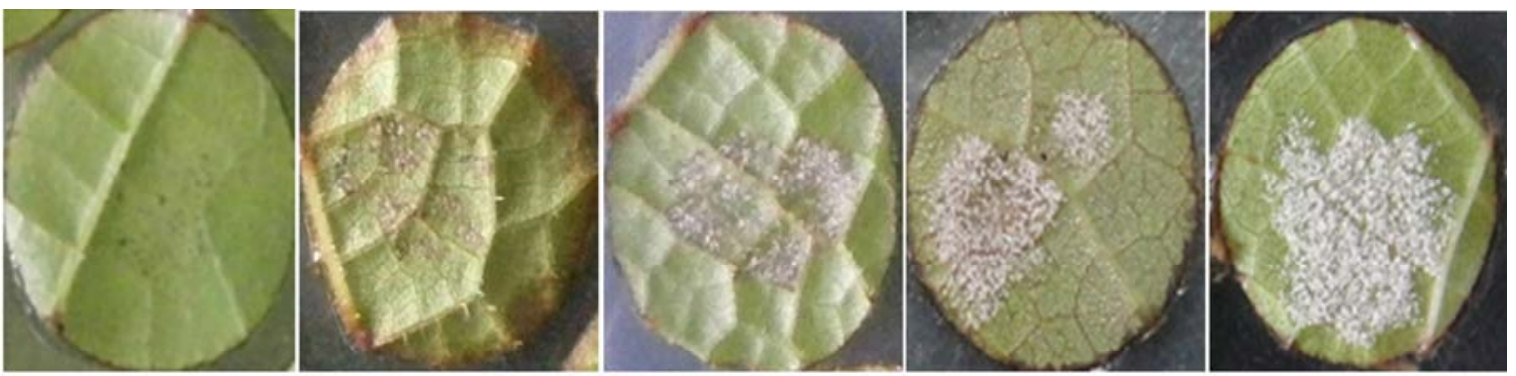

\section{Sporulation}

density

Fig. 1. Downy mildew symptoms on leaf discs with different sporulation density observed at 9 days postinoculation. 
(Table 1). In the species level, V. rotundifolia was immune to DM disease as no single disease incidence but only necrotic spots were observed among all the inoculations, while $V$. vinifera was the most susceptible species as indicated by the severity index (39.67 to $48.50 \%$ ) and sporulation density (3.73 to 4.00 ). Necrotic spots were also found on three Chinese Vitis species, V. hancockii, $V$. amurensis (except 'Tonghuasan'), and V. pseudoreticulata. Among the 10 Chinese Vitis species, eight (V. adenoclada, V. adstricta, V. amurensis, V. bellula, V. ficifolia, V. hancockii, V. pseudoreticulata, and $V$. quinquangularis) showed various degrees of resistance to DM disease (Table 1), and one of which, $V$. pseudoreticulata, showed extremely low disease incidence (2.5\%), distinguishing itself from the rest of them. The other two Chinese Vitis species, V. davidii and V. piasezkii, were classified as DM susceptible grape species due to their high severity index (47.33 and $37.75 \%)$ and high sporulation density scores (3.03 and 3.28). All $V$. amurensis cultivars exhibited low sporulation density (0.53 to 2.34), but their DM resistance levels varied. For example, $V$. amurensis 'Shuanghong' showed lower disease incidence $(53.33 \%)$ and severity index $(17.17 \%)$ than the other V. amurensis cultivars (Table 1). Both of the two VA-VV hybrids, 'Zuohongyi' and 'Zuoyouhong', were susceptible to DM disease with high disease incidence (100\%), severity index (47.78 and $45.75 \%)$, and sporulation density (3.70 and 3.77).

While all the variables (disease incidence, severity index, and sporulation density) are considered, five levels of DM resistance were distinguished (Table 1; Fig. 2): (i) the three cultivars of $V$. rotundifolia were "immune" to DM disease; (ii) V. pseudo-

TABLE 1. Score of downy mildew symptoms and sporulation 9 days after Plasmopara viticola infection

\begin{tabular}{|c|c|c|c|c|c|c|c|c|c|}
\hline \multirow{2}{*}{$\frac{\text { Species }}{\text { Vitis rotundifolia }}$} & \multirow{2}{*}{$\begin{array}{l}\text { Cultivars/accessions } \\
\text { Carlos }\end{array}$} & \multicolumn{2}{|c|}{ Disease incidence $(\%)^{\mathrm{y}}$} & \multicolumn{2}{|c|}{ Severity index $(\%)^{\mathrm{y}}$} & \multicolumn{2}{|c|}{ Sporulation density } & \multirow{2}{*}{$\begin{array}{l}\text { Resistant levels } \\
\text { Immune }\end{array}$} & \multirow{2}{*}{$\begin{array}{c}\begin{array}{c}\text { Necrotic } \\
\text { spots }^{\mathrm{z}}\end{array} \\
+\end{array}$} \\
\hline & & 0.00 & $\mathrm{D}$ & 0.00 & $\mathrm{E}$ & 0.00 & $\mathrm{~F}$ & & \\
\hline & Fry & 0.00 & $\mathrm{D}$ & 0.00 & $\mathrm{E}$ & 0.00 & $\mathrm{~F}$ & Immune & + \\
\hline & Noble & 0.00 & $\mathrm{D}$ & 0.00 & $\mathrm{E}$ & 0.00 & $\mathrm{~F}$ & Immune & + \\
\hline V. pseudoreticulata & $1057 / 1058$ & 2.50 & $\mathrm{D}$ & 0.58 & $\mathrm{E}$ & 0.03 & $\mathrm{~F}$ & Extremely resistant & + \\
\hline V. adenoclada & Mix & 39.29 & $\mathrm{C}$ & 4.47 & $\mathrm{DE}$ & 0.39 & $\mathrm{~F}$ & Resistant & - \\
\hline V. adstricta & 1008 & 82.50 & $\mathrm{AB}$ & 18.63 & $\mathrm{C}$ & 0.90 & $\mathrm{DEF}$ & Resistant & - \\
\hline V. bellula & 1104 & 63.57 & $\mathrm{ABC}$ & 22.14 & $\mathrm{BC}$ & 1.49 & $\mathrm{CDE}$ & Resistant & - \\
\hline$V$. ficifolia & 0943/0946 & 82.50 & $\mathrm{AB}$ & 22.65 & $\mathrm{BC}$ & 1.93 & $\mathrm{CD}$ & Resistant & - \\
\hline V. hancockii & 945 & 39.87 & $\mathrm{C}$ & 8.33 & $\mathrm{CDE}$ & 0.48 & $\mathrm{EF}$ & Resistant & + \\
\hline V. quinquangularis & Mao grape & 65.83 & $\mathrm{ABC}$ & 17.33 & CD & 1.50 & $\mathrm{CDE}$ & Resistant & - \\
\hline \multirow[t]{6}{*}{ V. amurensis } & Shuanghong & 53.33 & $\mathrm{BC}$ & 17.17 & $\mathrm{CD}$ & 0.53 & $\mathrm{EF}$ & Resistant & + \\
\hline & Changbaijiu & 100.00 & A & 36.81 & A & 2.34 & $\mathrm{BC}$ & Partially resistant & + \\
\hline & Shuangfeng & 98.33 & A & 38.83 & A & 1.65 & $\mathrm{CD}$ & Partially resistant & + \\
\hline & Shuangyou & 98.75 & A & 39.50 & A & 2.24 & $\mathrm{BC}$ & Partially resistant & + \\
\hline & Tonghuasan & 100.00 & A & 33.30 & $\mathrm{AB}$ & 1.68 & $\mathrm{CD}$ & Partially resistant & - \\
\hline & Zuoshanyi & 93.00 & A & 35.90 & A & 1.79 & $\mathrm{CD}$ & Partially resistant & + \\
\hline \multirow[t]{2}{*}{ VA-VV hybrids } & Zuohongyi & 100.00 & A & 45.75 & A & 3.70 & A & Susceptible & - \\
\hline & Zuoyouhong & 100.00 & A & 47.88 & A & 3.77 & A & Susceptible & - \\
\hline V. davidii & $940 / 941$ & 100.00 & A & 47.33 & A & 3.03 & $\mathrm{AB}$ & Susceptible & - \\
\hline V. piasezkii & $0957 / 0958$ & 96.67 & A & 37.75 & A & 3.28 & A & Susceptible & - \\
\hline \multirow{4}{*}{ V. vinifera } & Cabernet Sauvignon & 93.33 & A & 39.67 & A & 3.73 & A & Susceptible & - \\
\hline & Chardonnay & 99.17 & A & 39.67 & A & 3.87 & A & Susceptible & - \\
\hline & Thompson Seedless & 98.33 & A & 44.61 & A & 3.78 & A & Susceptible & - \\
\hline & Yatomi Rosa & 100.00 & A & 48.50 & A & 4.00 & A & Susceptible & - \\
\hline
\end{tabular}

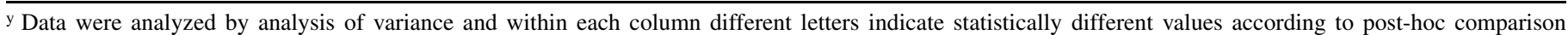
(Student Newman Keuls) at $P=0.05$.

${ }^{\mathrm{z}}+$, observed necrosis spots on leaf disc by $P$. viticola infection; and -, observed no necrosis spots on leaf disc by $P$. viticola infection.

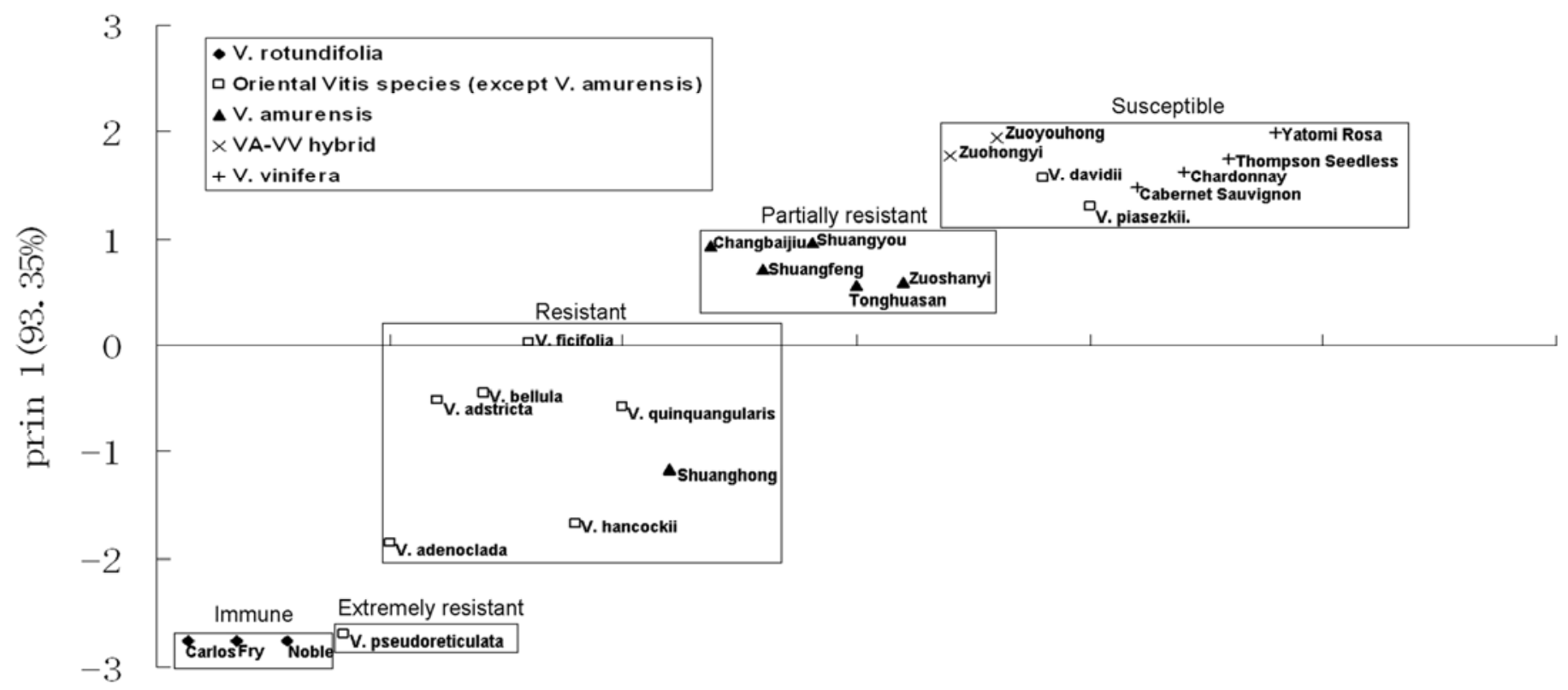

Fig. 2. Principal components analysis of all the recorded variables for downy mildew resistance among different grapevines investigated. 
reticulata '1057/1058' showed significantly lower scores of disease symptom than the rest of Euvitis species and were classified as "extremely resistant"; (iii) V. amurensis 'Shuanghong' and six other Chinese Vitis species (V. adenoclada, V. adstricta, V. bellula, V. ficifolia, V. hancockii, and V. quinquangularis) showed "moderate resistance" to $P$. viticola; (iv) five $V$. amurensis cultivars, 'Changbaijiu', 'Shuangfeng', 'Shuangyou', 'Tonghuasan', and 'Zuoshanyi', developed DM symptom but showed low sporulation density and were classified as "partly resistant"; and (v) two Chinese Vitis species (V. davidii and V. piasezkii), two VAVV hybrids ('Zuohongyi' and 'Zuoyouhong'), and four cultivars of $V$. vinifera ('Cabernet Sauvignon', 'Chardonnay', 'Thompson Seedless', and 'Yatomi Rosa') had similarly high disease incidence, severity index, and sporulation density, which were classified as "susceptible" to DM disease.

Microscopy studies of $\boldsymbol{P}$. viticola-grapevines interaction. In order to assess the responses of different Vitis species/cultivars to the pathogen attack, we compared the development of $P$. viticola

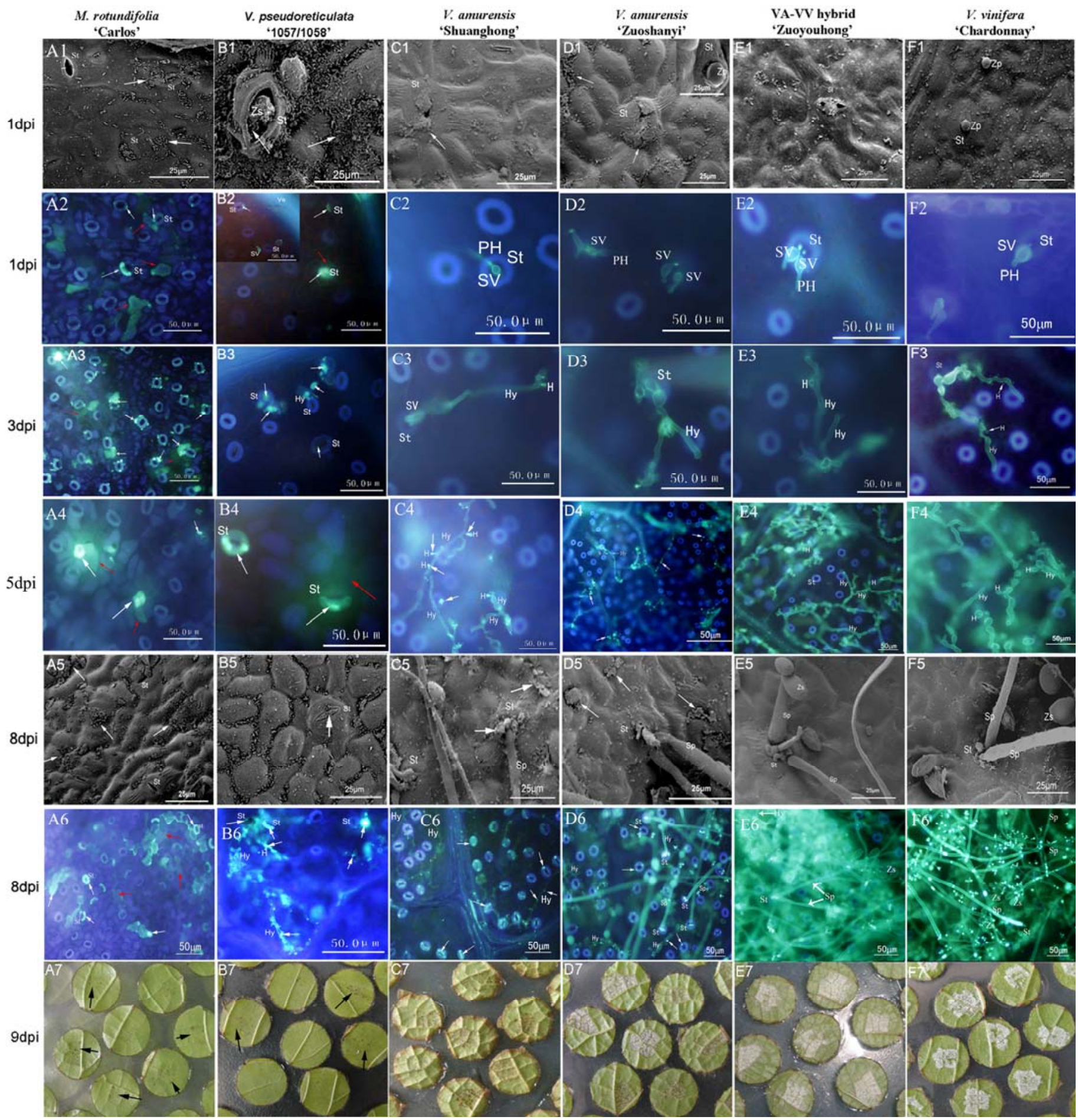

Fig. 3. Scanning electron microscopy (SEM) and fluorescence microscopy observations of Plasmopara viticola-infected leaves of Vitis species with different resistant levels at 1, 3, 5, and 8 days postinoculation (dpi). A, Immune $V$. rotundifolia 'Carlos'; B, extremely resistant $V$. pseudoreticulata '1057/1058'; C, resistant $V$. amurensis 'Shuanghong'; D, partially resistant $V$. amurensis 'Zuoshanyi'; E, susceptible VA-VV hybrid 'Zuoyouhong'; F, susceptible $V$. vinifera 'Chardonnay'; $\mathbf{1}$ and 5, SEM observations on the bottom of leaves at 1 and $8 \mathrm{dpi} ; \mathbf{2 , 3 , 4}$ and $\mathbf{6}$, fluorescence microscopy observations on the bottom of leaves at 1, 3, 5, and $8 \mathrm{dpi}$; and 7, macroscopic observations on leaf discs at 9 dpi. Black arrow, necrotic spot; gray arrow, distinctive fluorescence; white arrow, callose deposits; H, haustoria; Hy, hypha; PH, primary hypha; Sp, sporangiophore; St, stomata; SV, substomatal vesicle; Zp, zoospore; and Zs, zoosporangium. 
on the two immune $V$. rotundifolia cultivars ('Carlos' and 'Noble'), one extremely resistant V. pseudoreticulata (accession '1057/1058'), two DM resistant V. amurensis cultivars ('Shuanghong' and 'Zuoshanyi'), two susceptible VA-VV hybrids ('Zuohongyi' and 'Zuoyouhong'), and three susceptible $V$. vinifera cultivars ('Chardonnay', 'Thompson Seedless', and 'Yatomi Rosa'). At 1 dpi, zoospores were observed on all studied species by scanning electronic microscopy. They targeted on the guard cells of stomata, formed a germ tube, and penetrated through the stomatal opening. The number and size of zoospores at this stage were not different among these Vitis species. However, accumulations were observed in and around some of the infected stomata of DM immune $V$. rotundifolia (Fig. 3A1), extremely resistant $V$. pseudoreticulata (Fig. 3B1), and resistant and partly resistant $V$. amurensis cultivars (Fig. 3C1 and 3D1). These accumulations were previously reported as callose deposits $(21,53)$. On the other hand, no accumulation was observed in the stomata of susceptible VA-VV hybrid and $V$. vinifera grapevines (Fig. 3E1 and 3F1).

$\mathrm{KOH}$-aniline blue staining and fluorescence microscopy were used for studying the development of $P$. viticola hyphae in grapevine leaves and the callose deposits with highly yellow fluorescence intensity $(15,16,53)$. In DM immune $V$. rotundifolia (Fig. 3A2) and extremely resistant $V$. pseudoreticulata grapevines (Fig. 3B2), a strong yellow fluorescence observed near stomata indicated the possible presence of callose deposits. Cells with distinctive fluorescence were observed around the infected stomata. The substomatal vesicles (SV) with primary hyphae were observed in and around the stomata in all but the two $V$. rotundifolia cultivars. The percentage of stomata with SV observed in the extremely resistant V. pseudoreticulata $(5.45 \%)$ and resistant $V$. amurensis 'Shuanghong' (7.91\%) were much lower than that in partially resistant $V$. amurensis 'Zuoshanyi' (14.74\%), DM susceptible VA-VV hybrids 'Zuohongyi' (25.53\%) and 'Zuoyouhong' (14.56\%), and $V$. vinifera 'Chardonnay' (17.86\%), 'Thompson Seedless' (15.14\%), and 'Yatomi Rosa' (28.40\%, Table 2). The SVs in the more resistant grapevines were also smaller than in the susceptible ones based on the microscopy observation (Fig. 3B2 to F2).

At 3 dpi, no hypha but a lot of bright fluorescent spots, which were recognized as callose deposits, were found near the stomata in immune $V$. rotundifolia grapevines. Some infected cells of $V$. rotundifolia grapevines around the stomata developed a distinctive UV fluorescence (Fig. 3A3). In the extremely resistant $V$. pseudoreticulata grapevines, small unbranched hyphae and callose deposits in and around stomata were also observed at this stage (Fig. 3B3). In all other cultivars, the hyphae were elongated

TABLE 2. Development of Plasmopara viticola on Vitis species with different resistance levels by fluorescence microscopy observations

\begin{tabular}{|c|c|c|c|c|c|}
\hline \multirow{3}{*}{$\frac{\text { Species }}{V . \text { rotundifolia }}$} & \multirow{3}{*}{$\begin{array}{l}\text { Cultivars/accessions } \\
\text { Carlos }\end{array}$} & \multirow{2}{*}{\multicolumn{2}{|c|}{$\begin{array}{c}1 \mathrm{dpi}^{\mathrm{x}, \mathrm{y}} \\
\text { Stomata with } \\
\mathrm{SV}(\%)\end{array}$}} & \multirow{2}{*}{\multicolumn{2}{|c|}{$\begin{array}{c}3 \mathrm{dpi}^{\mathrm{y}, \mathrm{z}} \\
\text { Branched } \\
\text { hypha (\%) }\end{array}$}} \\
\hline & & & & & \\
\hline & & 0.00 & $\mathrm{D}$ & 0.00 & $\mathrm{C}$ \\
\hline & Noble & 0.00 & $\mathrm{D}$ & 0.00 & $\mathrm{C}$ \\
\hline V. pseudoreticulata & $1057 / 1058$ & 5.45 & $\mathrm{C}$ & 0.00 & $\mathrm{C}$ \\
\hline \multirow[t]{2}{*}{ V. amurensis } & Shuanghong & 7.91 & $\mathrm{C}$ & 6.25 & $\mathrm{C}$ \\
\hline & Zuoshanyi & 14.74 & $\mathrm{BC}$ & 54.05 & B \\
\hline \multirow[t]{2}{*}{ VA-VV hybrids } & Zuohongyi & 25.53 & $\mathrm{AB}$ & 55.17 & B \\
\hline & Zuoyouhong & 14.56 & $\mathrm{BC}$ & 58.33 & B \\
\hline \multirow[t]{3}{*}{ V. vinifera } & Chardonnay & 17.86 & $\mathrm{AB}$ & 66.67 & B \\
\hline & Thompson Seedless & 15.14 & $\mathrm{BC}$ & 58.33 & B \\
\hline & Yatomi Rosa & 28.40 & A & 90.00 & A \\
\hline
\end{tabular}

$\overline{\mathrm{x}}$ Number of stomata with substomatal vesicles (SV)/total number of stomata observed at 1 day postinoculation (dpi).

${ }^{y}$ Data were analyzed by analysis of variance and within each column different letters indicate statistically different values according to post-hoc comparison (Student Newman Keuls) at $P=0.05$.

${ }^{z}$ Number of branched hypha/total number of hypha at $3 \mathrm{dpi}$. and small swollen structures were formed around the tip of the hyphae, which were the early indication of haustoria formation. Fewer branched hyphae were observed in resistant $V$. amurensis 'Shuanghong' (6.25\%) than in partially resistant $V$. amurensis 'Zuoshanyi' (54.05\%), susceptible VA-VV hybrids 'Zuohongyi' (55.17\%) and 'Zuoyouhong' (58.33\%), and V. vinifera grapevines 'Chardonnay' (66.67\%), 'Thompson Seedless' (58.33\%), and 'Yatomi Rosa' (90.00\%, Table 2, Fig. 3C to F3).

At $5 \mathrm{dpi}$, there were significant differences in colonization of $P$. viticola in the leaf tissue among the cultivars/accessions investigated. In the DM immune $V$. rotundifolia and the extremely resistant $V$. pseudoreticulata grapevines, callose deposits and distinctive fluorescence cells were clearly observed (Fig. 3A4 and 3B4). Whereas in resistant $V$. amurensis cultivars, branched hyphae with small haustoria appeared, and some of the haustoria showed high fluorescence due to the callose deposits (Fig. 3C4 and 3D4). The callose deposits observed in V. amurensis cultivars were less abundant than that found in $V$. rotundifolia and $V$. pseudoreticulata cultivars. These DM resistant $V$. amurensis grapevines slowed down but could not completely stop the growth of $P$. viticola. Among them, less hyphae and haustoria were found in more resistant $V$. amurensis 'Shuanghong' than that in other partially resistant $V$. amurensis cultivars. In the mean time, the intercostal fields were almost filled with a complex mycelium with many haustoria in susceptible VA-VV hybrids and $V$. vinifera cultivars (Fig. 3E4 and 3F4).

At $8 \mathrm{dpi}$, callose deposits and cells with distinctive fluorescence were also clearly evident on DM immune $V$. rotundifolia (Fig. $3 \mathrm{~A} 5$ and 3A6). No hyphae were found in V. rotundifolia, while some abnormal hyphae of $P$. viticola were observed in $V$. pseudoreticulata (Fig. 3B6). Callose deposits were also observed in and around the stomata of $V$. pseudoreticulata and $V$. amurensis cultivars (Fig. 3B5-B6, C5-C6, and D5-D6), but these accumulations could not completely stop the emerging of sporangiophores. $P$. viticola sporangiophores were developed on cultivars of $V$. amurensis, V. vinifera, and VA-VV hybrids. The histological studies (fluorescence microscopy and scanning electron microscopy) revealed that more sporangiophores were found on VA-VV hybrids (Fig. 3E6) and V. vinifera cultivars (Fig. 3F6) than those on $V$. amurensis cultivars. In resistant $V$. amurensis 'Shuanghong' (Fig. 3C6), for example, little sporangiophores were observed.

Based on the microscopy observations, a schematic view of interaction between $P$. viticola pathogen and leaves in DM resistant and susceptible grapevines was summarized in Figure 4. In DM immune grapevines, callose deposits and distinctive fluorescence cells were induced by $P$. viticola infection, and the zoospores germination was inhibited in very early infection stage (Fig. 4A). In extremely resistant grapevines, callose deposits and distinctive fluorescence cells were also observed, but they could not completely inhibit the formation of $P$. viticola hyphae (Fig. 4B). In resistant grapevines, callose accumulated near the stomata in zoospore infection stage and around the haustoria in haustoria formation stage. It delayed but could not stop the growth of $P$. viticola (Fig. 4C). In partially resistant grapevines, the host responses were the same as resistant grapevines, but more hyphae were observed than that in resistant grapevines (Fig. 4D). In susceptible grapevines, no visible defense response was observed. A lot of hyphae grew into the intercellular space and rapidly colonized in the mesophyll tissues.

\section{DISCUSSION}

Many previous studies found that different grape species and cultivars displayed different responses to $P$. viticola infection $(5,12,50,52)$. The $V$. rotundifolia grapevines were recognized as having resistance to most of the grape diseases $(10,44)$, while $V$. vinifera was generally regarded as the most susceptible one $(4,5,8)$. Chinese wild Vitis species were found possessing 
moderate to high resistances to many diseases, including grape anthracnose, white rot $(39,57)$, downy and powdery mildew $(55,56)$. For example, V. pseudoreticulata, a Chinese Vitis species distributing in southeast of China, was highly resistant to powdery mildew (55). In our study, extremely resistant to downy mildew was also found in $V$. pseudoreticulata accession '1057/1058'. It appears that $V$. pseudoreticulata is the best source for breeding disease resistant grape cultivars among the Chinese Vitis species. However, contradictory reports were found about the DM resistance of $V$. amurensis. Some studies recognized that $V$. amurensis cultivars were susceptible to DM disease due to their high severity index $(49,55)$, while others recognized $V$. amurensis as DM resistant grapevines $(48,58)$. In this study, we found that although most of the $V$. amurensis cultivars showed high severity

A Immune

\section{zoospores infection and}

primary hypha formation

(1dpi) hypha formation

(3dpi) hypha elongation and

haustoria formation

(5dpi)

B

\section{Extremely Resistant}
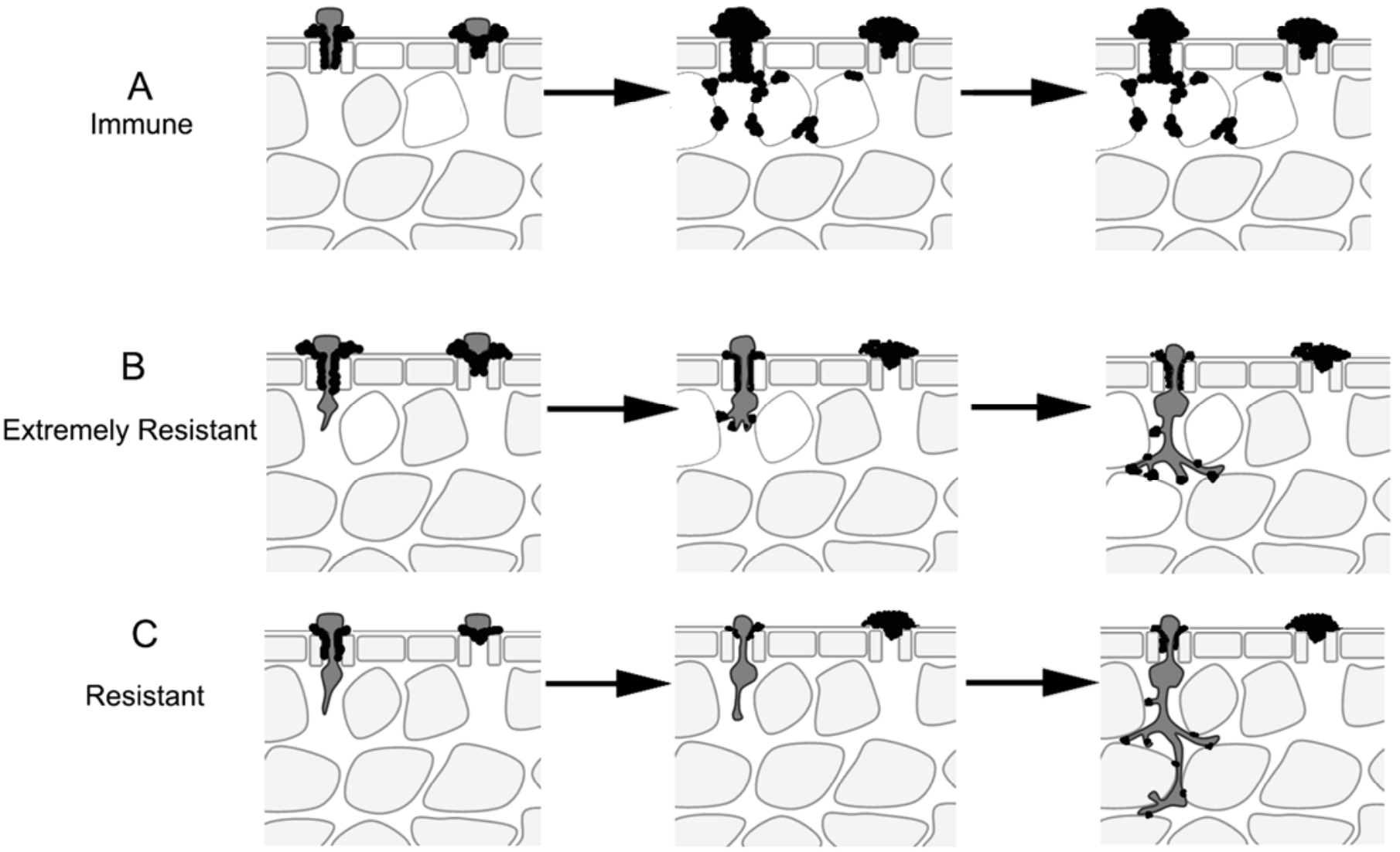

C

Resistant
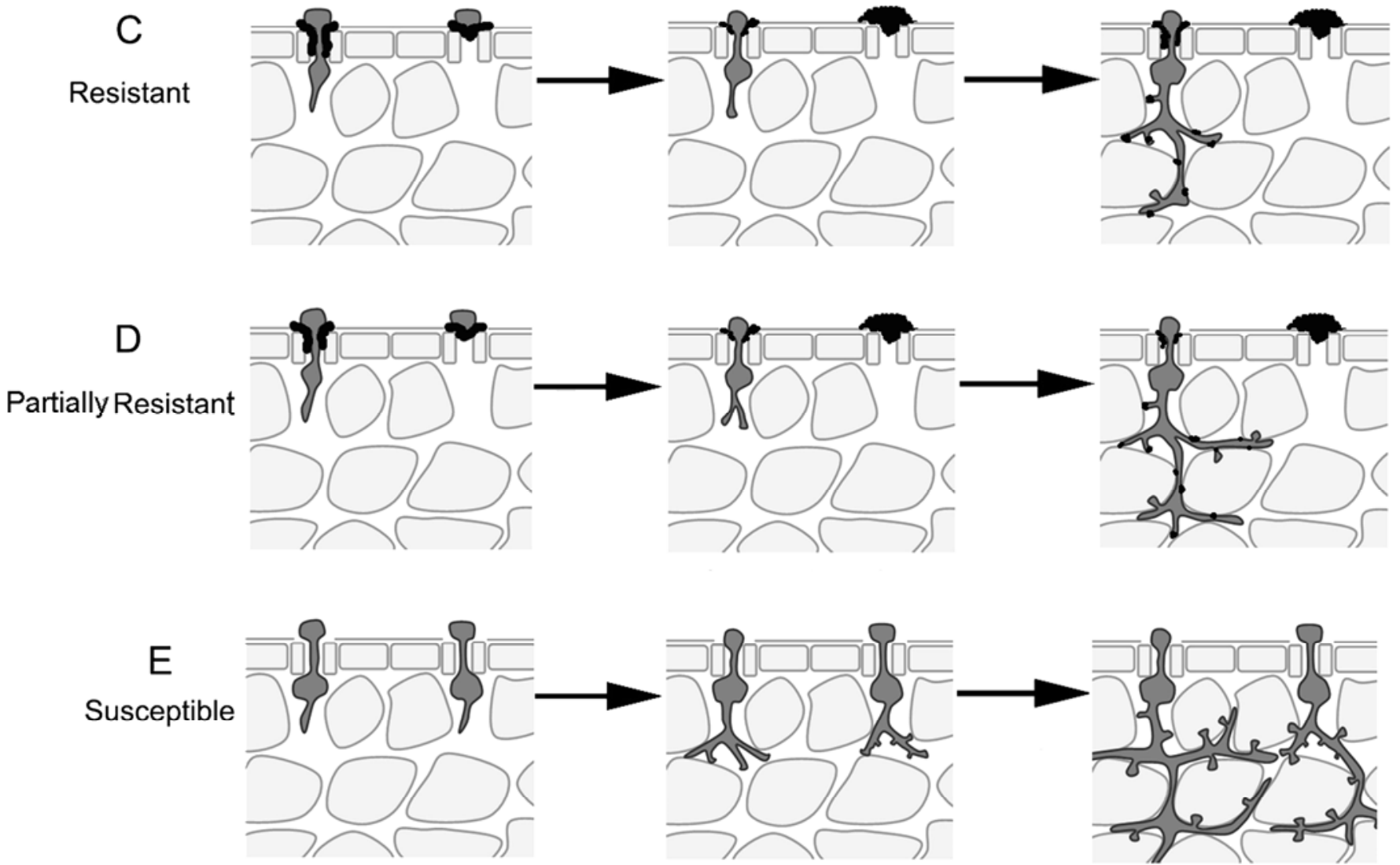

Fig. 4. Illustration of interaction between the Plasmopara viticola and grapevines with different downy mildew (DM) resistant levels: A, DM immune grapevine,

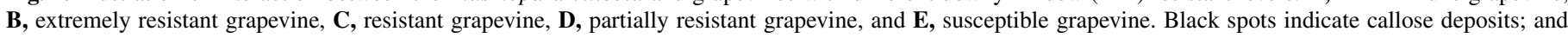
white cells indicate cells with distinctive fluorescence. 
index, their sporulation densities were much lower than DM susceptible $V$. vinifera grapevines, indicating at least partial resistance existed among these $V$. amurensis grapevines. Thus, more accurate assessment will be obtained when all three DM indicators (disease incidence, severity index, and sporulation density) are considered. For example, V. amurensis 'Shuanghong' was categorized as resistant to DM disease because of low score in each of the three indicators, while the other $V$. amurensis cultivars were considered as partly DM resistant grapevines according to their low score in just one of the indictors (sporulation density). The two cultivars derived from $V$. amurensis- $V$. vinifera crosses, 'Zuohongyi' [( $V$. amurensis 'Zuoshaner' $\times$ $V$. vinifera 'Мускат розовый' $) \times(V$. amurensis ' 73121 ' $\times V$. amurensis 'Shuangqing')] and 'Zuoyouhong' [(V. amurensis 'Zuoshaner' $\times V$. vinifera 'Мускат розовый' $) \times(V$. amurensis '73134' $\times$ V. amurensis 'Shuangqing')] $(43,47)$, were found susceptible to the DM disease, similar to the DM susceptible $V$. vinifera parents.

Leaf disc test is a suitable method to value the resistance and susceptibility of grapevines to $P$. viticola $(4,5,50)$. Our preliminary research (data not shown) also demonstrated that the DM resistance level was highly correlated between excised leaf disc and seedlings in the greenhouse. Meanwhile, we have also tested the DM resistance levels among grapevines cultivated in different locations (data not shown) and found that their DM resistant/susceptible status were fairly consistent.

The infection of $P$. viticola to grapevines proceeds when zoospores of $P$. viticola form germ tubes in stomata and then develop substomatal vesicles with primary hyphae. The hyphae of $P$. viticola extend into the intercellular spaces of the spongy parenchyma, where they develop an extensive mycelial network by forming haustoria that could penetrate into the cell walls of the host (9). Unger et al. (54) recognized that the main differences between resistant and susceptible grapevines appeared in the stage of hypha elongation and haustoria formation. In this study, however, the differences were observed in all stages post $P$. viticola infection, including zoospore infection, primary hypha formation, hypha elongation, haustoria formation, and sporangiophores emerging. The stages of zoospore infection and haustoria formation were the key stages of separating DM resistant and susceptible grapevines.

Callose deposition was recognized as one of the defense responses of plant to pathogen infection $(17,40,45)$. It is believed that callose deposits are typically triggered by conserved pathogen-associated molecular patterns and work as a mechanical barrier to limit pathogen growth $(7,24,51)$. In our study, callose deposits were observed in DM immune $V$. rotundifolia and extremely resistant $V$. pseudoreticulata grapevines at all observed time points. They were also found in the stomata and around the haustoria to a lesser degree in the less resistant $V$. amurensis grapevines, while no callose deposits were found in the DM susceptible grapevines. Similar observations were reported by Gindro et al. (21) when different levels of DM resistant $V$. vinifera cultivars were compared. In addition, Díez-Navajas et al. (16) reported that callose deposits were also deposited at the sites of haustoria formation in nonhost species infected by $P$. viticola. Callose deposits were also found in grapevines resistant to powdery mildew infection (28).

Cells with distinctive fluorescence were observed around the infected stomata in immune $V$. rotundifolia and extremely resistant $V$. pseudoreticulata grapevines. This fluorescence may have been caused by the accumulation of phenolics (2), and lignin-like deposits produced by peroxidase mediated cross-linking $(3,19,25)$. Additional histochemical tests at different wavelengths, however, are necessary to accurately confirm current observations. We also observed the local necrosis in immune $V$. rotundifolia, extremely resistant $V$. pseudoreticulata, resistant $V$. hancockii, and resistant/partially resistant $V$. amurensis grapevines by macroscopic observation. These responses may be associated with hypersensitive reactions $(11,40)$. Similar phenomenon were observed in grapevines infected by E. necator (18), lettuce infected by Bremia lactucae (38), and cruciferous infected by Hyaloperonospora parasitica (40). In our study, both the DM immune and highly resistant grape cultivars displayed a hypersensitive-like reaction, a typical response to pathogen infection in resistant plants (35). The dead mesophyll cells prevented the colonization and proliferation of the pathogen and can be accompanied by the accumulation of some phenolics, some of which acted as phytoalexins $(22,37,41,42)$. These responses can be part of the resistance responses and may be the key mechanism against $P$. viticola infection in these highly DM resistant grapevines.

\section{ACKNOWLEDGMENTS}

This study was supported by the earmarked fund for Modern Agroindustry Technology Research System (grant no. nycytx-30-zy-05) and Chinese Universities Scientific Fund (grant nos. 2012RC019 and 2011JS117). We are grateful to the following individuals and institutes for providing grape seedlings or leaves to this study: J. Wang at China Agricultural University, C. Liu at Zhengzhou Fruit Research Institute, and Y. Huang at Guangxi Agricultural Academy. We also thank W. Xu at China Agricultural University and Y. Tian at the Institute of Genetics and Developmental of Biology, China Academy of Science, for allowing us to access their microscopy facility.

\section{LITERATURE CITED}

1. Alleweldt, G., and Possingham, J. V. 1988. Progress in grapevine breeding. Theor. Appl. Genet. 75:669-673.

2. Bennett, M., Gallagher, M., Fagg, J., Bestwick, C., Paul, T., Beale, M., and Mansfield, J. 1996. The hypersensitive reaction, membrane damage and accumulation of autofluorescent phenolics in lettuce cells challenged by Bremia lactucae. Plant J. 9:851-865.

3. Bolwell, G. P. 1993. Dynamic aspects of the plant extracellular matrix. Int. Rev. Cytol. 146:261-324.

4. Boso, S., and Kassemeyer, H. H. 2008. Different susceptibility of European grapevine cultivars for downy mildew. Vitis 47:39-49.

5. Boso, S., Martínez, M. C., Unger, S., and Kassemeyer, H. H. 2006. Evaluation of foliar resistance to downy mildew in different cv. Albarino clones. Vitis 45:23-27.

6. Boso, S., Santiago, J. L., and Martinez, M. C. 2004. Resistance of eight different clones of the grape cultivar Albariño to Plasmopara viticola. Plant Dis. 88:741-744.

7. Brown, I., Trethowan, J., Kerry, M., Mansfield, J., and Bolwell, G. P. 1998. Localization of components of the oxidative cross-linking of glycoproteins and of callose synthesis in papillae formed during the interaction between non-pathogenic strains of Xanthomonas campestris and French bean mesophyll cells. Plant J. 15:333-343.

8. Brown, M. V., Moore, J. N., Fenn, P., and Mcnew, R. W. 1999. Inheritance of downy mildew resistance in table grapes. J. Am. Soc. Hortic. Sci. 124:262-267.

9. Burruano, S. 2000. The life-cycle of Plasmopara viticola, cause of downy mildew of vine. Mycologist 14:179-182.

10. Conner, P. J. 2006. A century of Muscadine grape (Vitis rotundifolia Michx.) breeding at the University of Georgia. Acta Hortic. (ISHS) 827:481-484.

11. Dai, G. H., Andary, C., Mondolot-Cosson, L., and Boubals, D. 1995. Histochemical studies on the interaction between three species of grapevine, Vitis vinifera, $V$. rupestris and V. rotundifolia and the downy mildew fungus, Plasmopara viticola. Physiol. Mol. Plant Pathol. 46:177-188.

12. Davidson, L. C. 2008. Variation within and between Vitis spp. for foliar resistance to the downy mildew pathogen Plasmopara viticola. Plant Dis. 92:1577-1584

13. de Wit, P. J. G. M. 2007. How plants recognize pathogens and defend themselves. Cell Mol. Life Sci. 64:2726-2732.

14. Desaymard, P. 1968. Norations et methodes de notation eel phyopharmaci. Phytiatr. Phytopharm. 2:163-173.

15. Díez-Navajas, A. M., Greif, C., and Merdinoglu, D. 2007. Two simplified fluorescent staining techniques to observe infection structures of the oomycete Plasmopara viticola in grapevine leaf tissues. Micron 38:680683.

16. Díez-Navajas, A. M., Wiedemann-Merdinoglu, S., Greif, C., and Merdinoglu, D. 2008. Nonhost versus host resistance to grapevine downy 
mildew, Plasmopara viticola, studied at the tissue level. Phytopathology 98:776-780.

17. Dong, X., Hong, Z., Chatterjee, J., Kim, S., and Verma, D. P. S. 2008. Expression of callose synthase genes and its connection with Npr1 signaling pathway during pathogen infection. Planta 229:87-98.

18. Feechan, A., Kabbara, S., and Dry, I. B. 2011. Mechanisms of powdery mildew resistance in the Vitaceae family. Mol. Plant Pathol. 12:263-274.

19. Fry, S. C. 1986. Cross-linking of matrix polymers in the growing cell walls of angiosperms. Annu. Rev. Plant Physiol. 37:165-186.

20. Fung, R. W., Gonzalo, M., Fekete, C., Kovacs, L. G., He, Y., Marsh, E., McIntyre, L. M., Schachtman, D. P., and Qiu, W. 2008. Powdery mildew induces defense-oriented reprogramming of the transcriptome in a susceptible but not in a resistant grapevine. Plant Physiol. 146:236-249.

21. Gindro, K., Pezet, R., and Viret, O. 2003. Histological study of the responses of two Vitis vinifera cultivars (resistant and susceptible) to Plasmopara viticola infections. Plant Physiol. Biochem. 41:846-853.

22. Glazebrook, J., Zook, M., Mert, F., Kagan, I., Rogers, E. E., Crute, I. R., Holub, E. B., Hammerschmidt, R., and Ausubel, F. M. 1997. Phytoalexindeficient mutants of Arabidopsis reveal that Pad4 encodes a regulatory factor and that four pad genes contribute to downy mildew resistance. Genetics 146:381-392.

23. Godard, S., Slacanin, I., Viret, O., and Gindro, K. 2009. Induction of defence mechanisms in grapevine leaves by emodin- and anthraquinonerich plant extracts and their conferred resistance to downy mildew. Plant Physiol. Biochem. 47:827-837.

24. Gomez-Gomez, L., Felix, G., and Boller, T. 1999. A single locus determines sensitivity to bacterial flagellin in Arabidopsis thaliana. Plant J. 18:277-284.

25. Graham, M. Y., and Graham, T. L. 1991. Rapid accumulation of anionic peroxidases and phenolic polymers in soybean cotyledon tissues following treatment with Phytophthora megasperma f. sp. glycinea wall glucan. Plant Physiol. 97:1445-1455.

26. Greenberg, J. T., and Yao, N. 2004. The role and regulation of programmed cell death in plant pathogen interactions. Cell. Microbiol. 6:201-211.

27. Hamiduzzaman, M. M., Jakab, G., Barnavon, L., Neuhaus, J. M., and Mauch-Mani, B. 2005. Beta-aminobutyric acid-induced resistance against downy mildew in grapevine acts through the potentiation of callose formation and jasmonic acid signaling. Mol. Plant-Microbe Interact. 18:819-829.

28. Heintz, C., and Blaich, R. 1990. Ultrastructural and histochemical studies on interactions between Vitis vinifera L. and Uncinula necator (Schw.) Burr. New Phytol. 115:107-117.

29. Hijwegen, T. 1963. Lignification, a possible mechanisms of active response against pathogens. Neth. J. Plant Pathol. 69:314-317.

30. Hood, M. E., and Shew, H. D. 1996. Applications of $\mathrm{KOH}$ aniline blue fluorescence in the study of plant-fungal interactions. Phytopathology 86:704-708.

31. Hückelhoven, R., Fodor, J., Preis, C., and Kogel, K. 1999. Hypersensitive cell death and papilla formation in barley attacked by the powdery mildew fungus are associated with hydrogen peroxide but not with salicylic acid accumulation. Plant Physiol. 119:1251-1260.

32. Jeandet, P., Douillet-Breuil, A. C., Bessis, R., Debord, S., Sbaghi, M., and Adrian, M. 2002. Phytoalexins from the Vitaceae: Biosynthesis, phytoalexin gene expression in transgenic plants, antifungal activity, and metabolism. J. Agric. Food Chem. 50:2731-2741.

33. Jones, J. D. G., and Dang, J. F. 2006. The plant immune system. Nature 444:323-329.

34. Jürges, G., Kassemeyer, H. H., Dürrenberger, M., Düggelin, M., and Nick, P. 2009. The mode of interaction between Vitis and Plasmopara viticola Berk. \& Curt. Ex de Bary depends on the host species. Plant Biol. 11:886-898.

35. Kamoun, S., Huitema, E., and Vleeshouwers, V. G. A. A. 1999. Resistance to oomycetes: A general role for the hypersensitive response? Trends Plant Sci. 4:196-200.

36. Kiefer, B., Riemann, M., Büche, C., Kassemeyer, H. H., and Nick, P. 2002. The host guides morphogenesis and stomatal targeting in the grapevine pathogen Plasmopara viticola. Planta 215:387-393.

37. Koch, E., and Slusarenko, A. 1990. Arabidopsis is susceptible to infection by a downy mildew fungus. Plant Cell 2:437-445.

38. Lebeda, A., Sedlářová, M., Petřivalský, M., and Prokopová, J. 2008. Diversity of defence mechanisms in plant-oomycete interactions: A case study of Lactuca spp. and Bremia lactucae. Eur. J. Plant Pathol. 122:71-89.

39. Li, D., Wan, Y. Z., Wang, Y. J., and He, P. C. 2008. Relatedness of resistance to anthracnose and to white rot in Chinese wild grapes. Vitis 47:213-215.

40. Li, H., Ge, X., Han, S., Sivasithamparam, K., and Barbetti, M. J. 2010. Histological responses of host and non-host plants to Hyaloperonospora parasitica. Eur. J. Plant Pathol. 129:221-232.

41. Li, H., Kuo, J., Barbetti, M. J., and Sivasithamparam, K. 2007. Differences in the responses of stem tissues of spring-type Brassica napus cultivars with polygenic resistance and single dominant gene-based resistance, to inoculation with Leptosphaeria maculans. Can. J. Bot. 85:191-203.

42. Li, H., Sivasithamparam, K., Barbetti, M. J., and Kuo, J. 2004. Differences in the germination and invasion by ascospores and pycnidiospores of Leptosphaeria maculans on canola varieties with varying susceptibility to blackleg. J. Gen. Plant Pathol. 70:261-269.

43. Lu, J., Schell, L., and Ramming, D. W. 2000. Interspecific hybridization between Vitis rotundifolia and Vitis vinifera and evaluation of the hybrids. Acta Hortic. 528:479-486.

44. Lu, W. P., Wang, J., Song, R. G., Zang, Y. F., Shen, Y. J., Guo, Z. G., Liu, Z. H., and Liu, J. K. 2000. Breeding research for cold-resistant grape variety of 'Zuohong No.1'. Sino-Overseas Grapevine and Vine 1:13-14. (In Chinese)

45. Nishimura, M. T., Stein, M., Hou, B., Vogel, J. P., Edwards, H., and Somerville, S. C. 2003. Loss of a callose synthase results in salicylic aciddependent disease resistance. Science 301:969-972.

46. Paxton, J. D. 1981. Phytoalexins-A working redefinition. J. Phytopathol. 101:106-109.

47. Song, R. G., Lu, W. P., Guo, T. J., Liu, J. K., Lin, X. G., Shen, Y. J., Guo, Z. G., Zhang, B. X., and Yang, Y. M. 2005. First breeding report of new dry-red brewing grape variety 'Zuoyouhong'. Sino-Overseas Grapevine and Vine 3:13-16. (In Chinese).

48. Song, R. G., Lu, W. P., Wang, J., Shen, Y. J., Shi, G. W., Li, W., Liu, Y. Z., Chang, G. C., and Jin, T. F. 1999. Studies on the breeding of 'Shuanghong'-A new cultivar of $V$. amurensis Rupr. Special Wild Economic Animal Plant Res. 1:17-20 (Chinese).

49. Song, R. G., Zhang, Y. F., Li, C. Y., Lu, W. P., Qu, H. G., and Wang, J. 1998. Study of resistance to Plasmopara viticola disease in Vitis amurensis. North. Hortic. 2:47-49. (In Chinese).

50. Sotolár, R. 2007. Comparison of grape seedlings population against downy mildew by using different provocation methods. Not. Bot. Hort. Agrobot. Cluj. 35:1842-4309.

51. Soylu, E. M., Soylu, S., and Mansfield, J. W. 2004. Ultrastructural characterization of pathogen development and host responses during compatible and incompatible interactions between Arabidopsis thaliana and Peronospora parasitica. Physiol. Mol. Plant Pathol. 65:67-78.

52. Thomma, B. P., Eggermont, K., Penninckx, I. A., Mauch-Mani, B., Vogelsang, R., Cammue, B., and Broekaert, W. F. 1998. Separate jasmonate-dependent and salicylate-dependent defense response pathways in Arabidopsis are essential for resistance to distinct microbial pathogens. Proc. Natl. Acad. Sci. USA 95:15107-15111.

53. Trouvelot, S., Varnier, A.-L., Allègre, M., Mercier, L., Baillieul, F., Arnould, C., Gianinazzi-Pearson, V., Klarzynski, O., Joubert, J.-M., and Pugin, A. 2008. A $\beta-1,3$ glucan sulfate induces resistance in grapevine against Plasmopara viticola through priming of defense responses, including HR-like cell death. Mol. Plant-Microbe Interact. 21:232-243

54. Unger, S., Büche, C., Boso, S., and Kassemeyer, H. H. 2007. The course of colonization of two different Vitis genotypes by Plasmopara viticola indicates compatible and incompatible host-pathogen interactions. Phytopathology 97:780-786.

55. Wan, Y. Z., Schwaniniger, H., He, P. C., and Wang, Y. J. 2007. Comparison of resistance to powdery mildew and downy mildew in Chinese wild grapes. Vitis 46:132-136.

56. Wang, Y. J., Liu, Y., He, P. C., Che, J., Lamikanra, O., and Lu, J. 1995. Evaluation of foliar resistance to Uncinula necator in Chinese wild Vitis species. Vitis 34:159-164.

57. Wang, Y. J., Liu, Y., He, P. C., Lamikanra, O., and Lu, J. 1998. Resistance of Chinese Vitis species to Elsinoe ampelina (de Bary) Shear. HortScience 33:123-126.

58. Wu, J., Zhang, Y. L., Zhang, H. Q., Huang, H., Folta, K. M., and Lu, J. 2010. Whole genome wide expression profiles of Vitis amurensis grape responding to downy mildew by using Solexa sequencing technology. BMC Plant Biol. 10:234-249. 